F. Child Lang. 24 (1997), 767-779. Printed in the United Kingdom

(C) 1997 Cambridge University Press

\title{
Overregularization in English plural and past tense inflectional morphology: a response to Marcus (1995)*
}

\author{
VIRGINIA A. MARCHMAN \\ University of Texas at Dallas \\ KIM PLUNKETT \\ Oxford University \\ JUDITH GOODMAN \\ University of Missouri, Columbia \\ (Received 6 Fune 1996. Revised 2 February 1997)
}

\begin{abstract}
\end{abstract}
In a recent note, Marcus (1995) suggests that the rate of overregularization of English irregular plural nouns is not substantively different from that of English irregular past tense verbs. This finding is claimed to be in conflict with the predictions of connectionist models (Plunkett \& Marchman, I99I, I993) which are said to depend solely on the dominance of regular over irregular forms in determining overregulation errors. However, these conclusions may be premature given that Marcus averaged overregulation rates across irregular nominal forms that varied in token frequency and across samples representing a broad range of children's ages. A connectionist view would predict an interplay between type frequency and other item level factors, e.g. token frequency, as well as differences in the developmental trajectories of the acquisition of nouns and verbs. In this response, we briefly review longitudinal parental report data $(N=26)$ which indicate that children are significantly more likely to produce noun overregularizations than verb overregularizations across a prescribed age period $(\mathrm{I} ; 5$ to $2 ; 6)$. At the same time, these data also show that children are familiar with proportionately more irregular nouns than irregular verbs. These findings are consistent with the predictions of Plunkett \& Marchman (I99I,

[*] This research was supported by grants from the National Institutes of Health (5 R 29 DC 02292), the Economic and Social Research Council, UK, and the John T. and Catherine D. MacArthur Foundation. The authors wish to thank Jennifer Jahn-Samilo for testing subjects and entering data, as well as the parents and children who participated. Address for correspondence: Virginia A. Marchman, The University of Texas at Dallas, School of Human Development, P.O. Box 830688, GR 4I, Richardson, TX, 75083-0688, USA. e-mail:vamarch@utdallas.edu. 
I 993) in that the larger regular class affects the frequency of noun errors but also that familiarity with individual irregular nouns tends to reduce the likelihood of overregularizations. In contrast to the conclusion of Marcus (I995), the connectionist approach to English inflectional morphology provides a plausible explanation of the phenomenon of overregularization in both the English plural and past tense systems.

\section{INTRODUCTION}

The productive use of inflectional morphology traditionally has been taken as core evidence that language involves a specialized system of rules and symbols (e.g. Pinker, I 99 I). Much of the evidence for this conclusion comes from studies of the English past tense which report productive use of the /ed/ suffix even with irregular verbs (e.g. goed) and a U-shaped pattern of development that involves initial correct usage, the subsequent onset of errors, and finally a return to correct usage (e.g. Cazden, i 968; Kuczaj, ı 977; Marcus, Ullman, Pinker, Hollander, Rosen \& Xu, I992). Within most symbolic accounts of this sort, it is assumed that regulars are generated by a default 'rule' (e.g. past $\rightarrow$ stem $+/$ ed/) which results in the on-line generation of grammatically inflected forms whenever it is not 'blocked' by the successful retrieval of an independently stored irregular form (a 'blockingand-retrieval-failure' model). This model further assumes that regular and irregular forms are processed by distinct, modularly-organized and independent mechanisms (e.g. Pinker, i 99 I ; Marcus et al., I 992).

A dual-mechanism symbolic account has been challenged by work within a connectionist framework which offers a substantially different conceptualization of the phenomenon of overgeneralization within the English past tense and the nature of linguistic systems more generally (Rumelhart \& McClelland, I987; MacWhinney \& Leinbach, I 99 I ; Plunkett \& Marchman, I99I, I993; Daugherty \& Seidenberg, I992; Marchman, I 993; Hare, Elman, \& Daugherty, i 995; see Elman, Bates, Johnson, Karmiloff-Smith, Parisi \& Plunkett, I 996 for review). This alternative proposes that a single lexically-based constraint-satisfaction mechanism utilizes factors such as type and token frequency, patterns of phonological regularity, and incremental learning in order to organize the regular mappings in the context of the heterogeneous set of irregular ones. This singlemechanism account was first substantiated by the computational demonstration that two mechanisms were not REQUIRED to implement key aspects of the behavioural profile of children who were in the process of learning this inflectional system (Rumelhart \& McClelland, I987; Plunkett \& Marchman, I 99 I, I 993). Importantly, however, several specific hypotheses have been empirically validated in children (Marchman \& Bates, r 994; Marchman, in press) and adults (Daugherty \& Seidenberg, I992; Marchman \& Callan, 
I 995), and have been substantiated crosslinguistically and diachronically (Hare \& Elman, I 995 ; Bybee, I 995).

In a recent note in this Journal, Marcus (1995) extends the discussion of these two models to another inflectional system, the English plural. Although the English plural and past tense systems share many properties (e.g. phonologically conditioned suffixation, irregular alternatives involving steminternal vowel change and zero-marking), there are some key configurational differences. Specifically, in the past tense, about a hundred or so commonly used irregular forms (e.g. go $\rightarrow$ went; see $\rightarrow$ saw) coexist with many more verbs that undergo one of three basic types of suffixation (i.e. the regulars). The plural system, in contrast, is vastly over-represented by regular forms, boasting only a handful of irregular alternatives to the 'add a regular suffix' pattern. Some of these irregular nouns are likely to be familiar to children (e.g. tooth $\rightarrow$ teeth; mouse $\rightarrow$ mice), while others are clearly less frequent in the speech to and by children (e.g. louse $\rightarrow$ lice) and/or are associated with particular contexts (e.g. reindeer $\rightarrow$ reindeer).

Marcus claims that the difference in the proportion of regular to irregular types in the English plural and past tense systems is key to distinguishing the predictions of a symbolic versus connectionist model of the acquisition of inflectional morphology. According to Marcus ( I 995), connectionist models should overregularize irregular nouns at a higher rate than irregular verbs because of the overwhelming predominance of regular to irregular nouns. The symbolic approach, in contrast, is immune to the effects of the relative size of the regular class. On this view, the default regular process always applies unless an irregular is encountered. The number of regular and irregular forms in the language is irrelevant.

Employing techniques similar to those in Marcus et al. (I992), Marcus (r995) analysed the rate of overregularization of irregular nouns in free speech corpora for so children from the CHILDES database. The number and frequency of samples for each child ranged from six occasional samples over a period of 13 months to 2 ro samples taken weekly over two and a half years. The entire period covered from $\mathrm{i} ; 3$ to $5 ; 2$. All clear examples of plural tokens were included (see Marcus, I 995, p. 45 I, for a description of which irregular nouns were excluded from the analysis). The total number of tokens analysed per child ranged from five to 307. Overregularization rate was calculated as the proportion of overregularized irregular plural tokens out of the sum of correct and overregularized plural tokens produced across all samples for a given child.

Comparing the findings from this analysis to that in Marcus et al. (I992), Marcus concluded that the acquisition of English nominal and verbal inflectional morphology is similar (rather than different) in two key ways:

(I) Averaging across children and items, the rate of overregularization of English irregular plurals is comparable to the rate of overregularization of 
English irregular past tense forms (about $8 \%$ ). (But note that this rate increases to $12.4 \%$ if one excludes the three children who produced fewer than seven irregular plural tokens (mean for the remainder of the sample $=$ I 62.9, range $=62-307$ based on Table 2, p. 453). All three of these children had an overregularization rate of $\circ \%$.)

(2) Early in development, children undergo a period in which all irregular forms are used correctly, prior to the onset of overregularizations. Of the children who produced any plural overregularizations (seven of ro), all of them produced correct irregular plurals prior to the onset of erroneous forms.

Marcus claims that these findings challenge 'connectionist models which attribute overregularization largely to the attraction of irregulars to large numbers of regulars' (p. 457). Further, these data would appear to contradict the view that there is a link between size of regular verb vocabulary and overregularization errors in studies of real children (Marchman \& Bates, I 994). Marcus concludes that his findings are instead 'consistent with models such as the blocking-and-retrieval-failure model in which regular forms are produced by rule' (p. 453).

EXPLAINING OVERREGULARIZATIONS IN CONNECTIONIST NETS

To evaluate Marcus' conclusions, we review the explicitly stated predictions of the connectionist approach regarding the basis for overregularizations in connectionist networks. First, patterns of acquisition in the Plunkett \& Marchman ( I 99 I, I 993) models rest not only on the number of exemplars of a given pattern (type frequency) but also on how frequently a given form is encountered (token frequency):

'One of the main findings of this work has been that a default regularization process will emerge in a network when it is exposed to a suitably configured vocabulary for a sufficient number of training trials. The default regularization process is blocked when the network recognized verb stems which it has been taught are irregular. Two factors enable irregular verb stems to block the default regularization process: (a) their high token frequency and (b) their phonological similarity to other irregular verbs’ (Plunkett \& Marchman, i 993, p. 64).

Thus, while high type frequency of regular forms provides the network with the information it needs to abstract the regular pattern, the high token frequency of individual stem-past tense pairs is one factor serving to protect individual items from error. In extending the predictions to the English plural, it is crucial to examine the impact of type frequency on rates of overregularization in the context of information regarding the token frequencies of individual irregular nouns. Several studies have demonstrated a link between children's acquisition of specific lexical items and their 
frequency in the input (e.g. Huttenlocher, Haight, Bryk, Seltzer \& Lyons, I99 I ; Barrett, Harris \& Chasin, I99 I), and this factor plays a considerable role in determining rates of overregularization in connectionist networks.

Secondly, in the discussion of the origins of $U$-shaped development, Marcus claims that connectionist models demonstrate early correct performance because of 'the high percentage of vocabulary which is irregular in the early stages of training' (p. 449). This is not an accurate characterization of performance in these networks. Early correct performance in the networks (prior to the onset of overregularizations) results from the network learning a small number of forms - regardless of mapping type. When vocabulary size is small, correct usage derives from the network underexploiting the resources available, allowing the network to 'memorize' individual mappings. In effect, each mapping is treated as if it were orthogonal to all other mappings. Consequently, the various stem-past tense pairs are not subject to interference. This pattern of performance is not unique to Plunkett \& Marchman (1993), but also applies to the learning exhibited in Rumelhart \& McClelland (I987). At the same time, the particular type of generalization behaviour subsequently exhibited by these networks is dependent on learning a sufficient number of regular mappings, a relationship that has been substantiated in studies of real children (Marchman \& Bates, 1994). Thus, by incorporating key features of children's vocabulary acquisition, such as small initial vocabulary size, the phenomenon of early correct performance can be framed in terms of the network's underutilization of resources. The ONSET of errors depends upon learning a sufficient number of regular exemplars, leading to interference between conflicting types of stem-past tense mappings and erroneous output (for more discussion see Plunkett \& Marchman, I 993, I 996).

EVALUATING PLURAL AND PAST TENSE OVERREGULARIZATIONS IN CHILDREN

In this paper, we present a comparison of the acquisition of English plural and past tense forms in a longitudinal sample of twenty-six children, focusing on the reported usage of correct irregular and overregularized nouns and verbs. Each child's production of plural and past tense forms is sampled at the same five time points, over a period in which overregularization errors first appear and hence are likely to be salient to parents. We use data from a well-established parental report instrument, the MacArthur Communicative Development Inventory (CDI) (Fenson et al., I 993). While not perfect in all respects, parental report can be viewed to have advantages over other techniques of language sampling in that it taps into parents' knowledge of their child's productions across a variety of contexts. Further, we restrict our analysis to a set of commonly used irregular nouns and verbs that are likely to represent the first irregular forms learned by children. Although these 
items will naturally vary in frequency in the speech to and by children, all of the items are common words that are likely to be represented in spontaneous speech samples.

We first examine the frequency of reported overregularizations of irregular nouns versus irregular verbs in this longitudinal sample of children. Secondly, we look at indices of the general familiarity of these irregular nouns versus verbs at each time point. If irregular plural nouns are more familiar than irregular past tense verbs to young children, this advantage is likely to be derived from a high frequency of occurrence in the input (e.g. Huttenlocher et al., I99I) and has the potential to protect irregular nouns from overregularization. Thus, we would predict that the errors in the nominal system would not be as apparent as one might expect given that the nominal system is overwhelmingly dominated by regular forms.

METHOD

Subjects

Parental report data from the CDI: Words and Sentences (Fenson et al., I 993) for 26 children ( 16 males, Io females) were analysed at five points between the ages of $\mathrm{I} ; 2$ and $2 ; 6$. Children were recruited through a subject pool and through personal referrals. Parents of children in the subject pool responded to newspaper advertisements asking families if they wished to participate in a variety of developmental studies. The subjects represented a range of ethnic, racial, educational, and socioeconomic groups. Most of the children $(64 \%)$ were either first born or had no siblings in the same household. Children were enrolled in the study when they were between six and eight months of age. Although none of the children were exposed to a second langauge at the time at which they were recruited, some of the children had some exposure to a language other than English due to subsequent child-care arrangements.

\section{Procedure}

Participants in this study were taking part in a larger study of language acquisition. The component of this study relevant to the current analyses involved parental report data collected on a monthly basis from the ages of $0 ; 8$ to $2 ; 6$. The parental report forms were mailed to the child's family about one week before the child's monthly birthday. The children also had monthly laboratory assessments beginning at $\mathrm{r} ; \mathrm{O}$, and parents returned the forms when they brought their child to the lab. After the first month, the items checked on the returned form were filled in on the next month's form prior to sending it. This was intended to cut down on the time needed to complete the form. However, parents were permitted to remove items that had been filled in previously, and many did so if they no longer believed that their 
child knew an item. A subset of those data are analysed here; in particular, responses to the CDI at each of the following five 3 -month intervals: $1 ; 6$, I ; 9,$2 ; \circ, 2 ; 3$, and $2 ; 6$.

Responses from two sections of the CDI were analysed:

( I) Vocabulary checklist. A total of 680 words are presented alphabetically within 22 subsections (e.g. animals, vehicles, etc.) and parents are asked to indicate which of the words their child 'says.' Each of these items appears in its singular or stem form.

(2) Sentences and Grammar. Parents are asked to indicate if their child produces irregular nouns in their correct plural form (five items). Parents are also asked to report whether their child produces irregular verbs in their correct past tense form (20 items). A subsequent section asks parents to indicate if they 'have heard (their) child say recently' overregularized forms of irregular nouns ( 10 items) and verbs (33 items).

Taking data from these two sections of the CDI together, we identified a total of five irregular nouns (child, foot, man, mouse, tooth) and I 6 irregular verbs (blow, break, buy, drink, eat, fall, get, go, have, hear, hold, make, run, see, sit, take) which appear on the Vocabulary Checklist and on both parts of the Sentences \& Grammar section. All five of these irregular nouns are generally familiar to children in this age range, represented in at least five of the samples reported in Marcus (I995). All i 6 irregular verbs are also likely to be generally familiar to young children, occurring in at least four, and more typically nine or ı。, of the samples analysed in Marcus et al. (1992).

Following the strategy used in Marchman \& Bates (1994), we can determine what proportion of these items a given child is reported to produce in the singular or stem form on the checklist, in the correct irregular plural or past tense form, and/or in an overregularized version. More specifically, we computed the proportion of IRREGULAR NOUNS (of five possible) that each child was reported to produce on the Vocabulary checklist. These data provide an estimate of each child's familiarity with irregular nouns, regardless of whether they are used in any type of plural form. We then calculated the proportion of these nouns that each child was reported to use in the CORRECT IRREGUlar PLURAL (of five possible, children, feet, men, mice, teeth). For example, a child who knows all five irregular nouns ( $100 \%$ ), but who only produces two of them in their correct plural form would have a correct irregular rate of $40 \%$ for nouns. ${ }^{1}$

Finally, we computed an estimate of each child's production of OVERREGU-

[I] Since we were interested in whether children produce irregular nouns or verbs that they KNOW as correct irregular plural or past tense forms (see also Marchman \& Bates, I 994), we did not include those few nouns or verbs which were reported to be produced in a correct irregular form (e.g. feet), but not in the corresponding singular or stem form on the checklist (e.g. foot). This same strategy was applied to the calculation of estimates of overgeneralizations for nouns and verbs as well. 
LARIZED PLURALS for the irregular nouns that they are reported to know. ${ }^{2}$ The five irregular nouns appear in ten overregularized plural forms on the Sentences and Grammar section of the CDI: childrens, childs, feets, foots, mans, mens, mices, mouses, tooths, teeths. For each of the five nouns, we asked whether the parent reported either one or both overregularizations (e.g. childrens and/or childs). We then calculated the proportion of irregular nouns (of five possible) that the parent reported that their child knew and that they produced in any overregularized form. For example, a child who is reported to know all five irregular nouns on the checklist and is also reported to say tooths, teeths, and foots, would have an overregularized plural proportion score of $40 \%$ (i.e. two of five possible nouns are overregularized in some way).

For verbs, we computed analogous estimates of the proportion of IRREGULAR VERBS which a child is reported to produce on the Vocabulary checklist (of i 6 possible). These items also appeared as CORRECT IRREGULAR PAST TENSE forms on the Sentences and Grammar checklist (blew, broke, bought, drank, ate, fell, got, went, had, heard, held, made, ran, saw, sat, took). We thus computed the proportion of irregular verbs that the child is reported to know that they also are reported to produce as correct irregular past tense forms. Finally, the proportion of OVERREGULARIZED PAST TENSES was calculated using the following items: blewed, blowed, breaked, broked, buyed, drinked, dranked, eated, ate, falled, getted, gotted, goed, wented, haved, heared, holded, maked, runned, ranned, seed, sitted, satted, taked. We again calculated the number of irregular verbs (of I 6 possible) that the child knows and that the child is reported to produce on the Sentence and Grammar checklist in any overregularized form (e.g. eated or ated or both).

In summary, we examine the frequency with which children are reported to use correct and overregularized plural and past tense forms. Comparisons of the acquisition of the English nominal and verbal system can thus be made in terms of general tendencies observable in a single sample of children who are followed longitudinally across the age range during which the productive use of English inflectional morphemes first tends to appear.

RESULTS

In Figure I, we present the mean proportions of irregular nouns and verbs which children are reported to produce in an overregularized form. These patterns of responses were evaluated with a repeated measures multivariate

[2] Following Marcus (1995), we restrict our analyses to overregularizations of irregular nouns. However, it is well known that children will also produce errors on REGULAR nouns, generating forms such as blockses or shoeses. Several examples of these regular-overregularizations are included in the Sentences and Grammar section of the CDI: Words and Sentences. However, the source of these and other types of potential productivity (e.g. zero-marking) are beyond the scope of the current discussion. 


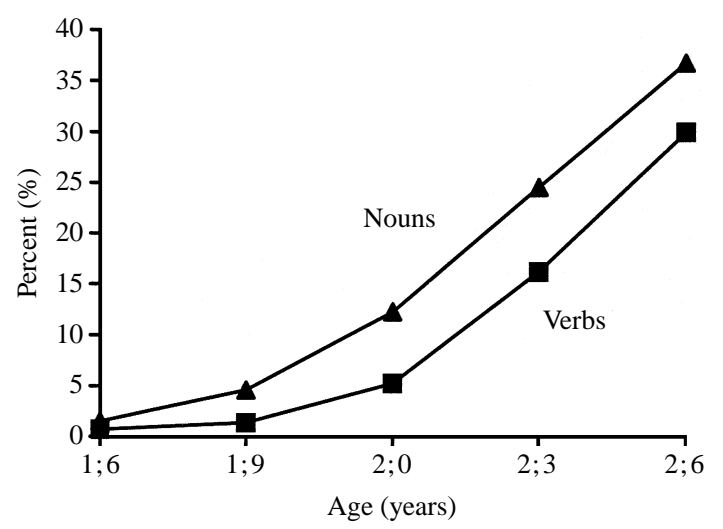

Fig. I. Proportion of irregular nouns and verbs reported on checklist and as overgeneralizations.

analysis of variance with item (noun vs. verb) and time (five levels) as withinsubjects factors. Not surprisingly, these data indicate that the proportion of overregularizations increased reliably across age for both nouns and verbs $(F(4,100)=24.66, p<0.001)$. However, in contrast to Marcus (1995), overregularized plurals $(M=\mathrm{I} 6.0 \%)$ were significantly more likely to be reported for these children than overregularized past tense forms of irregular verbs $(M=10.8 \%)(F(\mathrm{I}, 25)=5.2 \mathrm{I}, p<0.04)$. This effect is clearest after $2 ; 0$, prior to which children were rarely reported to produce any type of overregularization error. Four children were not reported to produce any overregularizations of either type at any time point.

Figure I also suggests that overregularized plural nouns were likely to be reported at a time point which is earlier than overregularized past tense verbs, however, no interaction between item type and time was observed across the entire sample. Yet, this interpretation is supported by the fact that $77.3 \%$ of the children ( 17 of the 22 who were reported to produce any overregularizations) were first reported to produce overregularized plurals at a point earlier $(N=9)$ or at the same time as $(N=8)$ their first reported productions of overregularized past tenses. Only five children were reported to produce overregularized past tense forms prior to the point at which their first overregularized plural form was reported. In general, these data suggest that the proportion of items for which children were reported to overregularize is reliably and consistently greater for nouns than verbs across the period studied here. In addition, there is some suggestion that errors on irregular nouns occurred developmentally prior to errors on irregular verbs.

Although children overregularized irregular nouns reliably more often than verbs, this tendency was not as great as one might predict given the quite substantial differences in the proportion of irregular types in the 


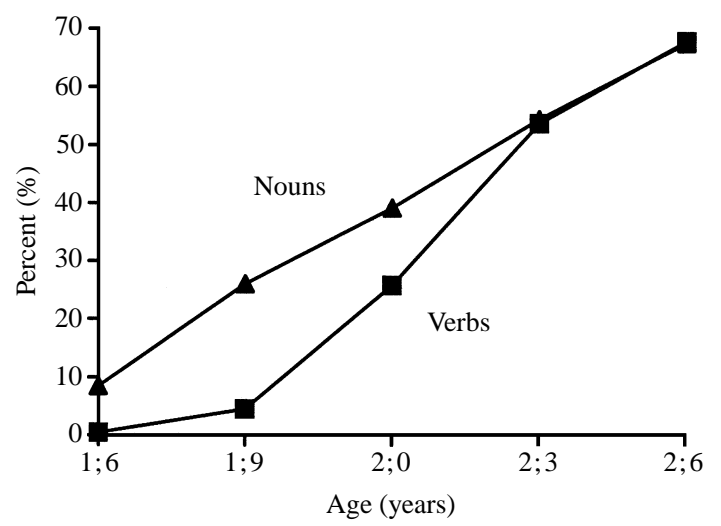

Fig. 2. Proportion of irregular nouns and verbs reported on checklist and in their correct irregular form.

English plural versus past tense systems. Thus, it seems reasonable to propose that some other factor might come into play in determining the onset and likelihood of overregularization errors. A connectionist approach would posit that items that are quite familiar to children would be resistant to overregularization errors.

Figure 2 presents the mean proportion of correct irregular forms of nouns and verbs reported for children at each of the five time points. These data indicate that correct irregular plural nouns $(M=39.2 \%)$ were indeed more likely to be included in the reported lexicons of these children than correct irregular past tense verbs $(M=30.6 \%)$. A repeated measures multivariate analysis of variance with time and item type (noun vs. verb) as the withinsubjects factors indicated that this difference is reliable $(F(\mathrm{I}, 25)=\mathrm{I} 6.63$, $p<0.00 \mathrm{I})$ across the entire time period, although clearly there is some convergence at the later assessment points. This convergence is reflected in a statistically reliable time by item interaction $\left(F(4, \mathrm{I} \circ 0)=5^{\circ} \circ 8, p<\right.$ ०.०० I $)$.

\section{DISCUSSION AND CONCLUSION}

These findings revealed reliable differences in the likelihood that parents will report that their child is overregularizing the English nominal and verbal regular inflections. Contrary to the findings reported in Marcus (I995), children were significantly more likely to be reported to produce overregularizations of nouns versus verbs during this period. Several factors may have contributed to the discrepancies between the two studies. First, Marcus ( 1995 ) averaged observations across an age range that went well beyond the period studied here. By focusing on a narrower age range, the current study suggests that at least one period of acquisition exists in which overregularized plural nouns are more likely to be observed than overregularized past tense 
verbs. Of course, this does not rule out the possibility that there are periods in which the converse is also the case, nor that the rates are generally comparable over the first five or so years of acquisition. However, in our view, collapsing across a broad and significant period of acquisition may have masked reliable and important distinctions in the developmental trajectories of the two systems.

Secondly, our analyses focused on standard sets of irregular nouns and verbs that are likely to comprise some of the earliest irregular forms that are acquired by children. By limiting our analyses to these subsets of items, we invariably risk losing access to the range of overregularization errors that are produced by these children across all irregular forms. At the same time, we gain insight into the patterns of overregularization that occur with respect to the forms that are most common in young children's lexicons and hence, that are the most likely to be reliably represented in naturalistic speech.

A final source of discrepancy is likely to rest in the use of parental report rather than naturalistic observation. There are clearly advantages and disadvantages of both classes of techniques (Fenson, Dale, Reznick, Bates, Thal \& Pethick, I 994; Tomasello \& Mervis, I 994), each with its own utility and benefits, as well as the potential for vulnerability to bias and sampling limitations. However, several recent studies have demonstrated that carefully constructed parental report instruments can serve as a reliable and valid technique for the study of several key phenomena of acquisition (Bates et al., I994; Marchman \& Bates, I994). Indeed, for this sample of children, rankorder correlations between size of reported production vocabulary from the $\mathrm{CDI}$ and performance on a laboratory word production task were quite high across most of the period analysed here (Jahn-Samilo, I 995). In light of these successes, the current study suggests that, at the very least, it was premature to accept the null hypothesis that meaningful and reliable differences in rate of overregularizations in the two systems do not exist.

These data also indicated that irregular plural forms are a familiar and well-established part of children's early lexicons, represented proportionately more frequently than irregular past tense forms in the lexicons of children in this age period. Children's familiarity with irregular plurals may allow those items to withstand interference from the larger class of regulars (i.e. resist regularization), as predicted by Plunkett \& Marchman (I99 I, I993). Of course, other factors are also proposed to play a role in the acquisition of individual items (e.g. phonological regularity), adding an additional constraint on the conditions for generalization and productive language use. We should emphasize that we have only touched on a subset of the factors that can possibly influence children's productive use of plural and past tense English inflectional morphology. In addition, it would be highly unlikely that acquisition in these domains progresses in isolation from each other or related systems, such as the English possessive. Modelling and empirical 
endeavours will no doubt continue to benefit from broadening the scope of inquiry beyond a single grammatical domain and exploring issues that arise crosslinguistically (e.g. Plunkett \& Nakisa, in press; Hare, Elman \& Daugherty, I995; cf. Marcus, Brinkman, Clahsen, Wiese, \& Pinker, I995; McCarthy \& Prince, I990).

In sum, within the connectionist perspective, the ability of a languagelearner to organize a heterogeneous set of mappings (e.g. regular and irregular forms) is dependent upon the competition and convergence among a variety of input-driven factors, each of which contribute to when a system will generalize and when the system is able to learn individual mapping pairs.

Based on models of only a small piece of children's grammatical systems, Plunkett \& Marchman (I99I, I993) suggested that type frequency was one important factor in determining patterns of productivity for children learning the English past tense. Subsequent analyses have suggested that this factor may indeed go a long way in predicting conditions for generalization across languages (see discussions in Bybee, I 995). However, adequate evaluations of the impact of type frequency, and thus the validity of the conclusions of Plunkett \& Marchman (I99I, I993) and others, cannot be made outside the context of other potentially relevant facts of grammatical systems, like token frequency. This short study sought to be a reminder of the importance of evaluating multiple sources of constraint on children's acquisition of inflectional morphology (see also Elman et al., i 996). Contrary to the conclusions drawn by Marcus ( ( 995), the results indicate that a connectionist viewpoint can offer a plausible account of the facts of acquisition for both the English plural and past tense systems.

\section{REFERENCES}

Bates, E., Marchman, V., Thal, D., Fenson, L., Dale, P., Reznick, J. S., Reilly, J. \& Hartung, S. (I994). Developmental and stylistic variation in the composition of early vocabulary. Fournal of Child Language 2I (I), 85-I24.

Barrett, M., Harris, M. \& Chasin, J. (I99I). Early lexical development and maternal speech: a comparison of children's initial and subsequent uses of words. Fournal of Child Language I8, 2 I-40.

Bybee, J. (1 995). Regular morphology and the lexicon. Language and Cognitive Processes ro (5), 425-55.

Cazden, C. B. (1968). The acquisition of noun and verb inflections. Child Development 39, $433-48$.

Daugherty, K. \& Seidenberg, M. S. (1992). Rules or connections? The past tense revisited. Proceedings of the I4th Annual Meeting of the Cognitive Science Society. Hillsdale, N.J.: Erlbaum.

Elman, J. L., Bates, E. A., Johnson, M. H., Karmiloff-Smith, A., Parisi, D. \& Plunkett, K. ( I996). Rethinking innateness : a connectionist perspective on development. Cambridge, MA: MIT Press.

Fenson, L., Dale, P. S., Reznick, J. S., Bates, E., Thal, D. \& Pethick, S. (I 994). Variability in early communicative development. Monographs of the Society for Research in Child Development 59 (5, Serial No. 242). 
Fenson, L., Dale, P. S., Reznick, J. S., Thal, D., Bates, E., Hartung, J., Pethick, S. \& Reilly, J.S. (I993). User's Guide and Technical Manual for the MacArthur Communicative Development Inventories. San Diego, CA. : Singular Press.

Hare, M. \& Elman, J. L. ( I 995). Learning and morphological change. Cognition 56 ( I), 6 I-98.

Hare, M., Elman, J. L. \& Daugherty, K. G. (I 995). Default generalization in connectionist networks. Language and Cognitive Processes ro (6), 60 I-30.

Huttenlocher, J., Haight, W., Bryk, A., Seltzer, M. \& Lyons, T. (r 99 I). Early vocabulary growth: relation to language input and gender. Developmental Psychology 27, 236-48.

Jahn-Samillo, J. (I995). Comprehension and production in children from 8 through 30 months of age: a comparison of parent report and laboratory measures. Paper presented at the Biennial Meeting of the Society for Research in Child Development (SRCD), Indianapolis, IN, March-April, I 995.

Kuczaj, S. A. (I977). The acquisition of regular and irregular past tense forms. Fournal of Verbal Learning and Verbal Behavior 16, 589-600.

MacWhinney, B. \& Leinbach, J. ( I 99 I). Implementations are not conceptualizations: revising the verb learning model. Cognition 40, I $2 \mathrm{I}-57$.

McCarthy, J. \& Prince, A. (I 990). Foot and word in prosodic morphology: the Arabic broken plural. Natural Language and Linguistic Theory 8, 209-83.

Marchman, V. A. (in press). Children's productivity in the English past tense: the role of frequency, phonology and neighborhood factors. Cognitive Science.

Marchman, V. A. (I993). Constraints on plasticity in a connectionist model of the English past tense. Fournal of Cognitive Neuroscience 5 (2), 2 I 5-34.

Marchman, V. A. \& Bates, E. ( I 994). Continuity in lexical and morphological development: a test of the critical mass hypothesis. Fournal of Child Language $2 \mathbf{r}$ (2), 33 I-66.

Marchman, V. A. \& Callan, D. (I995). Multiple determinants of the productive use of the regular past tense suffix. Proceedings of the I 7 th Annual Cognitive Science Society. Hillsdale, N.J.: Erlbaum.

Marcus, G. F. (I995). Children's overregularization of English plurals: a quantitative analysis. Fournal of Child Language $\mathbf{2 2}$ (2), 447-6o.

Marcus, G. F., Brinkman, U., Clahsen, H., Wiese, R. \& Pinker, S. ( I 995). German inflection: the exception that proves the rule. Cognitive Psychology 29 (3), I 89-256.

Marcus, G. F., Ullman, M., Pinker, S., Hollander, M., Rosen, T. J. \& Xu, F. (r992). Overregularization in language acquisition. Monographs of the Society for Research in Child Development 57 (4), Serial No. 228.

Pinker, S. (I99I). Rules of language. Science 253, 530-5.

Plunkett, K. \& Marchman, V. A. (I99I). U-shaped learning and frequency effects in a multilayered perceptron: implications for child language acquisition. Cognition $3^{8}$ (I), 43-102.

Plunkett, K. \& Marchman, V. A. (I 993). From role learning to system building: acquiring verb morphology in children and connectionist nets. Cognition 48 ( I), 2 I-69.

Plunkett, K. \& Marchman, V. A. ( I 996). Learning from a connectionist model of the English past tense. Cognition 6r (3), 299-308.

Plunkett, K. \& Nakisa, R. C. (in press). A connectionist model of the Arabic plural system. Language and Cognitive Processes.

Rumelhart, D. \& McClelland, J. ( 1987 ). Learning the past tenses of English verbs: implicit rules or parallel processing. In B. MacWhinney (ed.), Mechanisms of language acquisition. Hillsdale, N.J.: Erlbaum.

Tomasello, M. \& Mervis, C. (I 994). The instrument is great, but measuring comprehension is still a problem. Monographs of the Society for Research in Child Development $\mathbf{5 9}$ (5, Serial No. 242), I 74-I 79 . 